\title{
Colangiopancreatografía retrógrada endoscópica con papilotomía de urgencia versus tratamiento conservador en pancreatitis aguda grave por cálculos biliares (APEC trial): un estudio aleatorizado multicéntrico
}

\author{
Magdalena Ruiz-Esquide S. ${ }^{1}$, Catalina Reyes P. ${ }^{1}$, Javier Rodríguez G. ${ }^{2}$, \\ Luis Antonio Díaz P. ${ }^{3}$, Arnoldo Riquelme P. ${ }^{3}$ y Fernando Pimentel M. ${ }^{2}$
}

Urgent endoscopic retrograde cholangiopancreatography with sphincterotomy versus conservative treatment in predicted severe acute gallstone pancreatitis (APEC): a multicentre randomised controlled trial

\section{Contexto}

La pancreatitis aguda (PA) es una de las patologías gastrointestinales agudas más frecuentes en los ingresos hospitalarios, con incidencia en aumento debido a la obesidad y prevalencia de colelitiasis ${ }^{1}$. En efecto, la PA genera aproximadamente 300.000 hospitalizaciones y más de 20.000 muertes anuales en Estados Unidos, con un gasto que alcanza los 2,2 billones de dólares ${ }^{1}$.

Los cálculos biliares causan más del $60 \%$ de las PA y se asocian a complicaciones como colangitis y falla multiorgánica ${ }^{1,2}$. Entre 15 y $25 \%$ de todos los pacientes con PA desarrollan PA grave (PAG) $)^{3,4}$. El manejo de la pancreatitis aguda biliar se basa principalmente en soporte con reposo digestivo, reposición de fluidos con cristaloides, nutrición precoz y analgesia, reservando procedimientos endoscópicos o quirúrgicos para el manejo de sus complicaciones ${ }^{5-7}$. La colangiopancreatografía retrógrada endoscópica (CPRE) urgente en PA ha demostrado un beneficio en pacientes con colangitis concomitante, y se postula que podría ser beneficiosa en pacientes con colestasia, pero sin colangitis ${ }^{5-7}$. En pacientes con PA biliar sin colangitis y sin colestasia significativa, la papilotomía de urgencia descomprime el tracto biliar y pancreático, lo que potencialmente mejoraría el curso de la enfermedad; sin embargo, no está claro el beneficio de la CPRE urgente en este escenario.

La falta de evidencia respecto al manejo invasivo podría radicar en que la mayoría de los estudios realizaron la CPRE tardíamente (hasta tres días luego de la admisión), perdiendo el potencial beneficio de una descompresión precoz. Además, no todos realizan una papilotomía de rutina, procedimiento que podría ser beneficioso en pacientes con microlitiasis o barro biliar $^{8,9}$.

A continuación se presenta un estudio que compara la CPRE con papilotomía de urgencia versus el tratamiento estándar de soporte en pacientes con PAG biliar sin colangitis.

\section{Pregunta clínica}

En pacientes con una potencial PAG de etiología biliar sin colangitis, ¿Es útil la CPRE con papilotomía de urgencia en reducir complicaciones o mortalidad?

Para responder a la pregunta clínica se discutirá el siguiente artículo de investigación: Schepers NJ, Hallensleben NDL, Besselink MG, Anten MPGF, Bollen TL, da Costa DW, et al. Urgent endoscopic retrograde cholangiopancreatography with sphincterotomy versus conservative treatment in predicted severe acute gallstone pancreatitis (APEC): a multicentre randomised controlled trial. Lancet 2020;396:167-76 ${ }^{10}$.

\section{Métodos}

\section{Características generales}

\section{Pacientes}

Se evaluaron 1.178 adultos en el servicio de urgencia con diagnóstico de PAB en 26 hospitales en Países Bajos, desde febrero de 2013 hasta marzo de 2017. Tras evaluar los criterios de inclusión y exclusión se incluyeron 230 pacientes (Tabla 1).
'Escuela de Medicina, Pontificia Universidad Católica de Chile. Santiago, Chile.

${ }^{2}$ Departamento de Cirugía Digestiva, Pontificia Universidad Católica de Chile. Santiago, Chile. ${ }^{3}$ Departamento de Gastroenterología, Pontificia Universidad Católica de Chile. Santiago, Chile.

Recibido: 26 de julio 2021 Aceptado: 31 de julio de 2021

\section{Correspondencia a:} Dr. Fernando Pimentel $\mathrm{M}$.

Departamento de Cirugía Digestiva Facultad de Medicina Pontificia Universidad de Chile.

Diagonal Paraguay 362, Santiago, Chile. E-mail: fpimentelm1@ gmail.co 


\section{Medicina Basada en la evidencia en Gastroenterología}

\section{Intervención}

Se crearon dos grupos:

A)CPRE con papilotomía urgente en las primeras 24 horas tras el ingreso al servicio de urgencias y las primeras 72 horas tras el inicio de síntomas $(\mathrm{n}=117)$.

B) Tratamiento conservador $(\mathrm{n}=113)$.

Ambos grupos fueron tratados con hidratación intravenosa, analgesia, nutrición enteral según necesidad, sonda gástrica en caso de vómitos y tratamiento de insuficiencia pancreática endocrina y exocrina. El manejo en unidades de cuidados intensivos y de las complicaciones fue establecido por el tratante. En el grupo B se realizó CPRE con papilotomía sólo en caso de colangitis, o electiva en pacientes recuperados del episodio de pancreatitis inicial que presentaron colestasia persistente o litiasis biliar retenida.

\section{Resultados evaluados (Outcomes)}

El outcome primario fue un resultado compuesto por complicaciones mayores o mortalidad dentro de 6 meses posteriores a la aleatorización. Complicaciones mayores fueron insuficiencia orgánica persistente, necrosis pancreática, colangitis bacteriana, neumonía, insuficiencia pancreática exocrina o endocrina. Los outcomes secundarios fueron la necesidad y duración de estadía en unidad de cuidados intensivos, estadía hospitalaria, readmisiones hospitalarias por patología litiásica biliar, calidad de vida y costos sociales por 180 días tras la aleatorización.

\section{Evaluación de la validez interna}

Diseño: Estudio clínico prospectivo aleatorizado controlado multicéntrico (26 hospitales en Países Bajos).

Aleatorización: Secuencia de aleatorización automática 1:1 mediante un módulo de aleatorización basado en la web, con aleatorización en bloques variables.

Análisis de las características basales de los grupos: Se exponen las características basales, sin diferencias significativas. La colestasia estuvo presente en $63(54 \%)$ de 117 pacientes en el grupo de CPRE urgente y en 67 (59\%) de 113 pacientes en el de tratamiento conservador.

Ciego: Sin ciego.

Seguimiento: 180 días (6 meses), sin pérdidas.

Interrupción precoz: No.

\section{Resultados}

No hubo diferencia significativa en el outcome primario (mortalidad o complicación mayor) entre CPRE precoz (38\%) y tratamiento conservador (valor $\mathrm{p}=0,370$ ) (Tabla 2). Respecto a los outcomes secundarios, se identificaron diferencias en el desarrollo de

Tabla 1. Criterios de inclusión y exclusión

Criterios inclusión
Edad $\geq 18$ años
PA grave de etiología biliar, ${ }^{\mathrm{a}, \mathrm{b}, \mathrm{c}}$
$\begin{aligned} & \text { En caso de un episodio previo de pancreatitis necrotizante, el pa- } \\ & \text { ciente tuvo que estar completamente recuperado (confirmado en } \\ & \text { imágenes) }\end{aligned}$
$\begin{aligned} & \text { Capacidad para realizar CPRE dentro de las } 24 \text { h posteriores a la } \\ & \text { presentación al departamento de emergencias y no más de } 72 \mathrm{~h} \\ & \text { después del inicio de los síntomas }\end{aligned}$

\section{Criterios inclusió}

Edad $>18$ años

\section{Criterios exclusión}

Colangitis $^{\mathrm{d}}$

PA etiología no biliar (alcohol, causas metabólicas, medicamentos, etc.)

INR que no se corrigiera a $<1,5$ con factores de coagulación o fresco plasma congelado

\section{Embarazo}

Papilotomía o precorte previo

Antecedente médico de pancreatitis crónica

${ }^{a}$ Diagnóstico de pancreatitis aguda ( 2 de 3 criterios): dolor hemiabdomen superior; Amilasa o Lipasa sobre tres veces el límite superior normal; diagnóstico imagenológico. ${ }^{\mathrm{b}} \mathrm{Gravedad}$ : APACHE $\geq 8$, Imrie $\geq 3$ o PCR $>150 \mathrm{mg} / \mathrm{L}$ dentro de las $24 \mathrm{~h}$ del ingreso. ${ }^{\mathrm{c} E t i o l o g i ́ a ~ b i l i a r: ~ p r e s e n c i a ~ d e ~}$ barro biliar - cálculos biliares y/o dilatación vía biliar ( $>8 \mathrm{~mm}$ en pacientes de $\leq 75$ años o $>10 \mathrm{~mm}$ en pacientes de $>75$ años) y/o ALT sobre dos veces el límite superior normal. ${ }^{\mathrm{d}}$ Diagnóstico de colangitis: $\mathrm{T}^{\circ} \geq 38,5^{\circ} \mathrm{C}$ con calofríos o $\mathrm{T}^{\circ} \geq 39^{\circ} \mathrm{C}$ sin calofríos, sin otra etiología evidente, además de cualquiera de los siguientes 3 criterios: coledocolitiasis (diagnóstico imagenológico en ecotomografía, tomografía, endosonografía o resonancia magnética); dilatación colédoco biliar ( $>8 \mathrm{~mm}$ en pacientes de $\leq 75$ años o $>10 \mathrm{~mm}$ en pacientes de $>75$ años); colestasia progresiva (durante al menos dos días consecutivos y una bilirrubina $>2,3 \mathrm{mg} / \mathrm{dL}$ ). 


\section{Medicina Basada en la evidencia en Gastroenterología}

Tabla 2. Impacto de la CPRE urgente en pacientes con potencial pancreatitis aguda grave en términos de mortalidad o complicación mayor

\begin{tabular}{|c|c|c|c|c|c|c|c|}
\hline $\begin{array}{l}\text { Outcome } \\
\text { principal }\end{array}$ & $\begin{array}{c}\text { RA Grupo } \\
\text { CPRE } \\
\text { n (\%) }\end{array}$ & $\begin{array}{c}\text { RA Grupo } \\
\text { conservador } \\
\text { n }(\%)\end{array}$ & Valor $\mathbf{p}$ & $\begin{array}{c}\text { RR } \\
(\text { IC 95\%) }\end{array}$ & RRR & RRA & NNT \\
\hline $\begin{array}{l}\text { Mortalidad o com- } \\
\text { plicación mayor }\end{array}$ & $45(38 \%)$ & $50(44 \%)$ & 0,37 & $\begin{array}{c}0,87 \\
(0,64-1,18)\end{array}$ & 0,14 & 0,06 & 17 \\
\hline
\end{tabular}

${ }^{a}$ Complicaciones mayores: falla orgánica persistente, necrosis del parénquima pancreático, bacteriemia, colangitis, neumonía o insuficiencia pancreática endocrina o exocrina; RA: riesgo absoluto; RR: riesgo relativo; RRR: reducción del riesgo relativo; RRA: reducción del riesgo absoluto; NNT: número necesario a tratar.

colangitis durante la hospitalización (10\% tratamiento conservador versus $2 \%$ CPRE precoz, $p=0,010)$, reingresos por PA biliar ( $9 \%$ tratamiento conservador versus $0 \%$ CPRE precoz, $p=0,001)$ y reingresos por coledocolitiasis $(6 \%$ tratamiento conservador versus $1 \%$ CPRE precoz, $\mathrm{p}=0,033$ ) (Tabla 3). De los 10 pacientes reingresados por PA biliar, cuatro eran colecistectomizados previamente y en seis no se habían realizado una colecistectomía durante la hospitalización inicial. No hubo diferencias en otros outcomes secundarios (Tabla 3).

En el grupo de CPRE urgente, 5 (4\%) de 117 pacientes no se sometieron a CPRE de urgencia. Por otro lado, en el grupo de tratamiento conservador $(\mathrm{n}=113)$ se realizó una CPRE en $35(31 \%)$ de los pacientes, debido a colangitis $(\mathrm{n}=13)$, colestasia persistente $(\mathrm{n}=21)$ y para instalación de endoprótesis $(n=1)$. Se realizaron 128 CPRE en el grupo de CPRE urgente y 44 en el grupo de tratamiento conservador (reducción absoluta 66\%). Se produjeron eventos adversos por la CPRE en 4 pacientes: $3(3 \%)$ en el grupo de CPRE urgente versus $1(3 \%)$ en el grupo de manejo conservador.

En un análisis del subgrupo de pacientes con colestasia al momento de la aleatorización $(\mathrm{n}=130)$, el outcome primario se produjo en 20 de 63 (32\%)

Tabla 3. Outomes secundarios principales

\begin{tabular}{|c|c|c|c|c|}
\hline Outcomes secundarios & $\begin{array}{l}\text { Grupo CPRE } \\
\quad(\mathrm{n}=117)\end{array}$ & $\begin{array}{l}\text { Grupo conservador } \\
\qquad(\mathrm{n}=113)\end{array}$ & RR (IC 95\%) & Valor $\mathbf{P}$ \\
\hline Mortalidad $^{a}$ & $8(7 \%)$ & $10(9 \%)$ & NS & 0,37 \\
\hline \multicolumn{5}{|l|}{ Complicación mayor: } \\
\hline - Insuficiencia orgánica ${ }^{a}$ & $22(19 \%)$ & $17(15 \%)$ & NS & 0,57 \\
\hline - Colangitis ${ }^{\mathrm{a}}$ & $2(2 \%)$ & $11(10 \%)$ & $0,18(0,04-0,78)$ & $0,010^{*}$ \\
\hline - Bacteremia ${ }^{a}$ & $17(15 \%)$ & $25(22 \%)$ & NS & 0,14 \\
\hline - Neumonía & $9(8 \%)$ & $10(9 \%)$ & NS & 0,75 \\
\hline - Necrosis Pancreática $\mathrm{a}^{\mathrm{a}, \mathrm{b}}$ & $17(15 \%)$ & $18(16 \%)$ & NS & 0,77 \\
\hline - Insuficiencia pancreática endocrina o exocrina ${ }^{a}$ & $9(8 \%)$ & $3(3 \%)$ & NS & 0,086 \\
\hline Estadía hospitalaria (días) $^{\mathrm{c}}$ & $13[9-24]$ & $14[10-26]$ & NS & 0,67 \\
\hline Ingreso a $\mathrm{UCI}^{\mathrm{a}}$ & $24(21 \%)$ & $13(12 \%)$ & NS & 0,063 \\
\hline $\begin{array}{l}\text { Readmisión por complicación relacionada a cálculos } \\
\text { biliares }^{\text {a }}\end{array}$ & $14(12 \%)$ & $24(21 \%)$ & NS & 0,058 \\
\hline - PA biliar recurrente & 0 & $10(9 \%)$ & NA & $\mathbf{0 , 0 0 1}{ }^{*}$ \\
\hline - Colecistitis & $10(9 \%)$ & $7(6 \%)$ & NS & 0,5 \\
\hline - Colangitis & $1(1 \%)$ & $3(3 \%)$ & NS & 0,36 \\
\hline - Cólico biliar & $4(3 \%)$ & $7(6 \%)$ & NS & 0,37 \\
\hline - Coledocolitiasis & $1(1 \%)$ & $7(6 \%)$ & $0,14(0,02-1,10)$ & $\mathbf{0 , 0 3 3}{ }^{*}$ \\
\hline Costo económico en euros & 24.627 & 24.595 & NS & 0,994 \\
\hline
\end{tabular}

${ }^{a}$ Datos presentados como número/ número total de pacientes (\%). ${ }^{b}$ Se realizó una tomografía computada con contraste tras 5-7 días de hospitalización. ${ }^{c}$ datos presentados como mediana [rango intercuartil]. *Significancia estadística. NS: diferencia no significativa. NA: no aplica. 
pacientes en el grupo de CPRE urgente, en comparación con 29 de 67 (43\%) pacientes en el de tratamiento conservador, sin diferencias significativas $(\mathrm{p}=0,180)$.

\section{Comentarios}

\section{Riesgo de sesgo}

Se realizó una aleatorización adecuada con un sistema web automatizado. No se mencionan diferencias significativas en las características basales de ambos grupos. La estratificación de gravedad de la pancreatitis fue estandarizada, sin embargo, el valor predictivo positivo de los sistemas de puntuación clínica es moderado, pudiendo estratificar como grave inicialmente a pacientes que evolucionan con una PA leve. Además, se pudo haber incluido pacientes con colangitis, pero se usaron criterios estrictos y tal sesgo si existiera estaría a favor de la CPRE urgente debido al efecto terapéutico establecido de la CPRE en colangitis, sin embargo no se encontraron diferencias entre los grupos, por lo que el efecto de la CPRE no se sobrestima por este sesgo potencial. Al ser la CPRE un procedimiento invasivo, ni los participantes ni los tratantes eran ciegos. Tampoco se menciona el número de pacientes en los que se canuló accidentalmente el conducto pancreático, y si en aquellos casos fue instalada una prótesis pancreática transitoria, ya que esta complicación podría afectar la interpretación de los resultados.

El diagnóstico de colestasia fue basado en la elevación de bilirrubina o dilatación de la vía biliar. Es llamativo que no se incluyera la elevación de fosfatasas alcalinas, especialmente ante las múltiples causas de hiperbilirrubinemia que podemos encontrar en pacientes con PAG.

\section{Resultados}

Los resultados son relevantes porque la CPRE con papilotomía de urgencia no demostró reducir el riesgo de complicaciones mayores o mortalidad; aunque hubo más casos de colangitis en el grupo conservador, no hubo impacto en la mortalidad. Al considerar la estrategia conservadora y uso de CPRE sólo en pacientes con colangitis o colestasia persistente, aproximadamente $66 \%$ de los pacientes no necesitaron la CPRE, evitando las complicaciones y costos asociados.

Con respecto a los reingresos, una colecistectomía durante la misma hospitalización podría haber evitado las PA biliares recurrentes en estos pacientes. Se realizó un análisis del subgrupo de pacientes con PAG biliar y colestasia; aunque teóricamente estos pacientes podrían beneficiarse más de la CPRE urgente, no se observó una diferencia significativa. Es importante considerar que este resultado podría haber sido obtenido por un error tipo II.

\section{Aplicabilidad externa}

Este es un tema muy relevante en nuestro país debido a la gran prevalencia de patología biliar. Un estudio reciente de Berger $\mathrm{Z}$ y cols. evidenció que el $64,4 \%$ de las PA en Chile son de etiología biliar (70\% mujeres y $52 \%$ hombres $)^{11}$. La heredabilidad de la patología litiásica biliar excede el $50 \%$ en población Hispánica, donde se han identificado múltiples variantes genéticas litogénicas, lo que contribuye enormemente a la carga de enfermedad por esta patología ${ }^{12,13}$. Aunque la CPRE de urgencia existe en nuestro país, está escasamente disponible con la rapidez descrita en este estudio. Por lo tanto, es necesario contar con mayor evidencia que sustente este procedimiento antes de plantear realizar CPRE en pacientes con una potencial PAG sin colangitis ni obstrucción biliar al ingreso hospitalario.

\section{Conclusiones}

La CPRE con papilotomía de urgencia no redujo las complicaciones mayores o mortalidad en pacientes con potencial PAG de etiología biliar sin colangitis, por lo que está indicada sólo en pacientes con colangitis o colestasia persistente. Con una estrategia conservadora y CPRE sólo en estos casos, alrededor de dos tercios de los pacientes se evitan un procedimiento invasivo.

\section{Referencias}

1. Cucher D, Kulvatunyou N, Green DJ, Jie T, Ong ES. Gallstone pancreatitis: a review. Surg Clin North Am 2014;94:257-80.

2. Hines OJ, Pandol SJ. Management of severe acute pancreatitis. BMJ 2019;367:16227.
3. Robert JH, Frossard JL, Mermillod B, Soravia C, Mensi N, Roth M, et al. Early prediction of acute pancreatitis: Prospective study comparing computed tomography scans, ranson, glasgow, acute physiology and chronic health evaluation II scores, and various serum markers.
World Journal of Surgery 2002;26:612-9. https://doi.org/10.1007/s00268-0010278-y.

4. Beger HG, Rau BM. Severe acute pancreatitis: Clinical course and management. World J Gastroenterol 2007;13:5043-51. 


\section{Medicina Basada en la evidencia en Gastroenterología}

5. Working Group IAP/APA Acute Pancreatitis Guidelines. IAP/APA evidence-based guidelines for the management of acute pancreatitis. Pancreatology 2013;13:e1-15.

6. Crockett SD, Wani S, Gardner TB, Falck-Ytter Y, Barkun AN, American Gastroenterological Association Institute Clinical Guidelines Committee. American Gastroenterological Association Institute Guideline on Initial Management of Acute Pancreatitis. Gastroenterology 2018;154:1096-101.

7. Baron TH, DiMaio CJ, Wang AY, Morgan KA. American Gastroenterological Association Clinical Practice Update: Management of Pancreatic Necrosis. Gastroenterology 2020;158:67-75.e1.

8. van Santvoort HC, Besselink MG, de
Vries AC, Boermeester MA, Fischer $\mathrm{K}$, Bollen TL, et al. Early endoscopic retrograde cholangiopancreatography in predicted severe acute biliary pancreatitis: a prospective multicenter study. Ann Surg 2009;250:68-75.

9. Tse F, Yuan Y. Early routine endoscopic retrograde cholangiopancreatography strategy versus early conservative management strategy in acute gallstone pancreatitis. Cochrane Database Syst Rev 2012:CD009779.

10. Schepers NJ, Hallensleben NDL, Besselink MG, Anten M-PGF, Bollen TL, Da Costa DW, et al. Urgent endoscopic retrograde cholangiopancreatography with sphincterotomy versus conservative treatment in predicted severe acute gallstone pancreatitis (APEC): a multicentre randomised controlled trial. Lancet 2020;396:167-76.

11. Berger Z, Mancilla C, Tobar E, Morales MP, Baró M, Carrasco M, et al. Acute pancreatitis in Chile: A multicenter study on epidemiology, etiology and clinical outcome. Retrospective analysis of clinical files. Pancreatology 2020;20:63743.

12. Gompertz M, Lara I, Fernández L, Miranda JP, Mancilla C, Watkins G, et al. Mortalidad de la pancreatitis aguda: experiencia de 20 años en el Hospital Clínico Universidad de Chile. Rev Med Chile 2013;141:562-7.

13. Lammert F, Gurusamy K, Ko CW, Miquel J-F, Méndez-Sánchez N, Portincasa P, et al. Gallstones. Nat Rev Dis Primers 2016;2:16024. 